\title{
Avaliação de problemas relacionados a medicamentos em pacientes polimedicados
}

\author{
Evaluation of drug-related problems in polymedicated patients
}

Recebido em: 02/08/2020 Aceito em: $26 / 02 / 2021$
Aline Aparecida Pereira SOUZA; Ana Carla BROETTO-BIAZON;

Tânia Pereira SALCI-ARAN

Centro Universitário Integrado de Campo Mourão. Rodovia BR 158, Km 207,

CEP: 87300-970. Campo Mourão, PR, Brasil.

E-mail: allineapsouza_@hotmail.com

\section{ABSTRACT}

Although the objective is to improve the quality of life, polymedication is directly associated with the increased likelihood that the patient will develop drug-related problems (DRP), thus compromising the treatment's effectiveness, besides generating risks for the treatment and health. Thus, the present study performed pharmacotherapeutic follow-up in polymedicated patients from a community pharmacy service. The present study aimed to carry out pharmacotherapeutic follow-up in polymedicated patients using a community pharmacy service through a prospective and quantitative study in a pharmacy school at Campo Mourão, PR, Brazil. Twenty-six patients with chronic diseases and being treated with five or more medications were included in the study. The pharmacotherapeutic follow-up was performed based on the methodology proposed by the Dáder method. Drugdex ${ }^{\circledR}$, Dynamed, and ANVISA's electronic files were used to evaluate the therapy. DRP were classified according to Rovers and Currie, and the Beers criteria were used to identify inappropriate drugs for the elderly. Most patients were elderly $(80.8 \%)$, with three or more diseases and on average use of eight medications. 108 DRP (mean: 4.9 DRP/patient) were observed, which resulted in 76 proposals for pharmaceutical interventions to patients. Among the identified DRP, those related to safety (40.7\%), effectiveness (35.2\%), and adverse reactions $(27.8 \%)$ were highlighted. Among the factors that can be pointed out as predisposing to the high incidence of PRM, polymedication, advanced age and the presence of chronic diseases stand out.

Keywords: pharmaceutical assistance; polymedication; health worker-patient relations; pharmacological treatment.

\section{RESUMO}

Apesar de ter como objetivo a melhora da qualidade de vida, a polimedicação está diretamente associada ao aumento da probabilidade de o paciente desenvolver problemas relacionados a medicamentos (PRM), podendo assim comprometer a eficácia e segurança do tratamento. Com isto, o presente estudo teve como objetivo realizar o seguimento farmacoterapêutico em pacientes polimedicados usuários de um serviço de farmácia comunitária. Trata-se de um estudo prospectivo, de caráter quantitativo, realizado em uma 
farmácia escola localizada na cidade de Campo Mourão, PR. Foram incluídos 26 pacientes portadores de doenças crônicas e em tratamento com cinco ou mais medicamentos. O seguimento farmacoterapêutico foi realizado com base na metodologia proposta pelo método Dáder. Foram utilizados o Drugdex ${ }^{\circ}$, o Dynamed e o bulário eletrônico da ANVISA para avaliar as terapias. Os PRM foram classificados de acordo com Rovers e Currie e os critérios de Beers foram utilizados para identificação de medicamentos inapropriados para idosos. A maioria dos pacientes eram idosos $(80,8 \%)$, portadores de três ou mais enfermidades e em uso contínuo de oito medicamentos em média. Foram observados 108 PRM (média: 4,9 PRM/paciente), os quais resultaram em 76 propostas de intervenções farmacêuticas aos pacientes. Dentre os PRM identificados destacaram-se os relacionados à segurança $(40,7 \%)$, efetividade $(35,2 \%)$ e reações adversas $(27,8 \%)$. Entre os fatores que podem ser apontados como predisponentes para a elevada incidência de PRM, destacam-se a polimedicação, a idade avançada e a presença de doenças crônicas.

Palavras-chave: assistência farmacêutica; polimedicação; relações profissional-paciente; tratamento farmacológico.

\section{INTRODUÇÃO}

Apesar da terapia farmacológica ter como objetivo a melhora da qualidade de vida dos pacientes, a polimedicação está diretamente associada ao aumento da probabilidade de o paciente desenvolver problemas relacionados a medicamentos (PRM). Não há uma definição consensual para o termo polimedicação, entretanto a mais comumente relatada é a utilização de cinco ou mais medicamentos por dia (1). Geralmente, a polimedicação é observada em idosos, devido a presença de doenças crônicas, alterações fisiológicas e/ou outras morbidades $(2,3)$. No entanto, tal prática pode estar associada à farmacoterapia de indivíduos de qualquer faixa etária, visto que a incidência de doenças crônicas e comorbidades tem crescido. Estima-se que $40 \%$ da população adulta brasileira apresente alguma doença crônica e 29,8\% seja acometida por duas ou mais doenças $(4,5)$.

Os PRM estão diretamente associados à quantidade de medicamentos utilizados pelo paciente e são capazes de comprometer a eficácia do tratamento farmacológico e/ou gerar problemas de saúde $(6,7)$. Estes eventos representam também um grave problema de saúde pública, visto que quando não identificados e revertidos a tempo, causam agravos à saúde, gerando assim custos adicionais decorrentes de consultas, hospitalizações, utilização de mais medicamentos e outros serviços, podendo até mesmo levar o paciente a óbito $(8,9)$.
Neste contexto, torna-se evidente a necessidade da inserção do acompanhamento farmacoterapêutico no tratamento desses pacientes. A prática clínica farmacêutica pode garantir ao paciente uma terapia adequada e segura a fim de se obter os resultados desejados, minimizando os riscos de desfechos negativos e complicações decorrentes do uso irracional dos medicamentos $(10,11)$.

O acompanhamento ou seguimento farmacêutico tem como finalidade avaliar a terapia farmacológica do paciente, identificando e prevenindo possíveis PRM. A documentação e mensuração de resultados, bem como a divulgação dos mesmos colaboram para difundir a prática de uma forma fundamentada. Assim, o presente estudo teve como objetivo realizar o seguimento farmacoterapêutico em pacientes polimedicados usuários de um serviço de farmácia comunitária.

\section{MÉTODO}

Tipo de estudo e caracterização da amostra. O presente trabalho refere-se a um estudo prospectivo, realizado no período de julho de 2016 a dezembro de 2017, em uma farmácia escola, localizada na cidade de Campo Mourão, PR. O estudo foi aprovado pelo comitê de ética do Centro Universitário Integrado de Campo Mourão sob CAAE $\mathrm{n}^{\circ}$ 58793216.5.0000.0092. 


\section{lutarma}

Figura 1. Fluxograma de seguimento farmacoterapêutico.

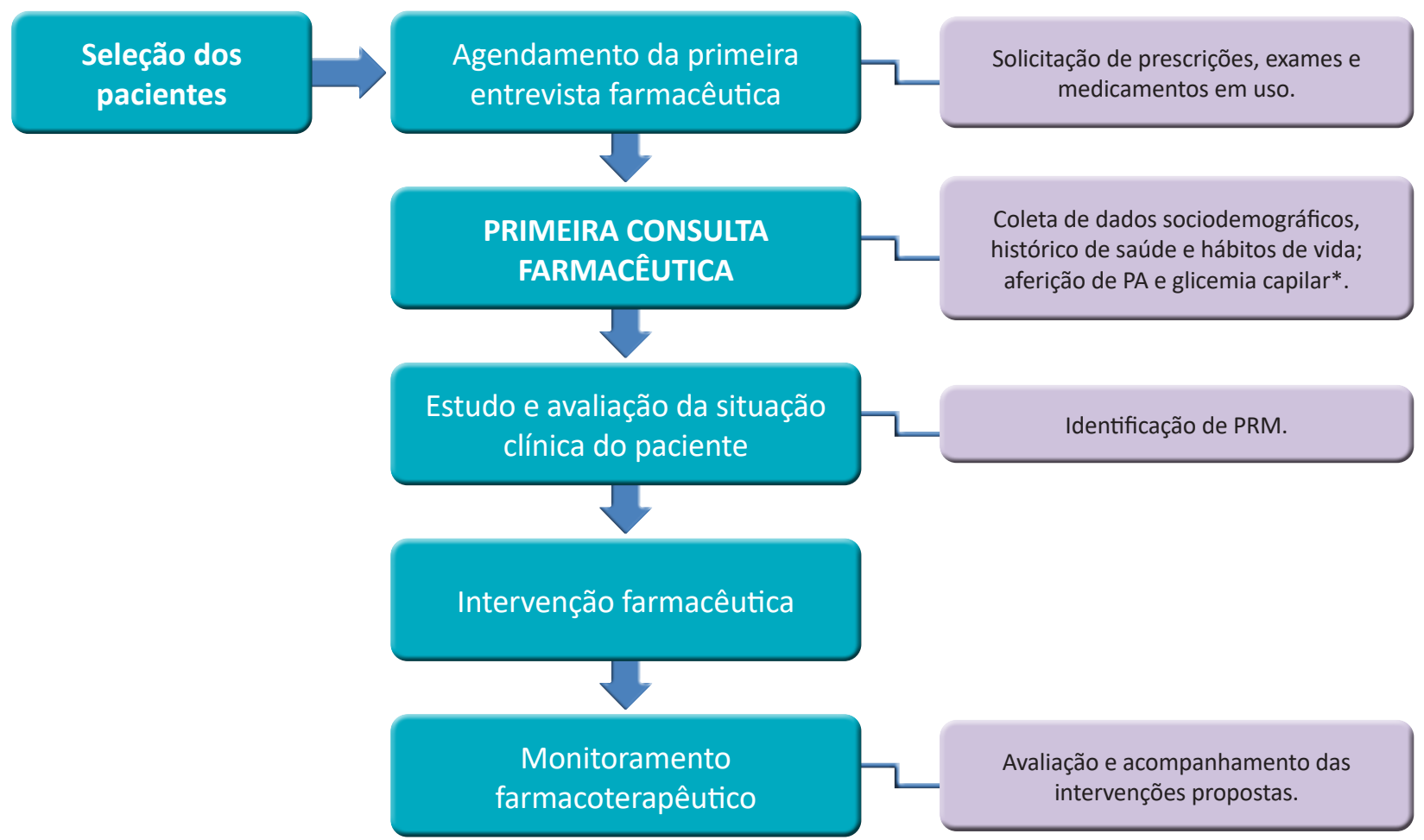

PRM: Problema relacionado a medicamento. $\left({ }^{*}\right)$ : Quando pertinente.

Foram incluídos indivíduos portadores de doenças crônicas e polimedicados, ou seja, com prescrição de cinco ou mais fármacos (15) e, usuários da farmácia escola. Os pacientes eram convidados a participar do seguimento e caso aceitassem, era agendada a data da primeira consulta, na qual solicitava-se que o paciente levasse as prescrições e últimos exames clínicos e laboratoriais (Figura 1)

Coleta de dados e seguimento farmacoterapêutico. A partir dos registros da farmácia escola, 32 pacientes foram selecionados para participar do estudo, dos quais 26 aceitaram. $\mathrm{Na}$ anamnese inicial foram coletadas informações referentes a situação clínica do paciente e sua terapia farmacológica.

Para tal, foi utilizada uma ficha adaptada do Método Dáder (6), com questões que abrangiam hábitos referentes à doença e ao histórico clínico do paciente, hábitos de vida, utilização dos medicamentos e sintomas apresentados. Durante a anamnese, foi realizada a aferição da pressão arterial (P.A.) (12) e, quando pertinente, realizado o teste de glicemia capilar, por meio de glicosímetro True
$\operatorname{Read}^{\circledR}$ (NIPRO, Sorocaba, Brasil), a fim de avaliar o controle glicêmico (13). Foram coletados dados antropométricos e calculados o índice de massa corporal (IMC) e a Razão Cintura/Quadril (RCQ) (14). A cada consulta, foi pedido para que os pacientes levassem os últimos exames laboratoriais.

A terapia farmacológica de cada paciente foi revisada a fim de avaliar a indicação terapêutica de cada medicamento prescrito, posologia recomendada, possíveis reações adversas, precauções e interações medicamentosas presentes na terapia.

As interações medicamentosas foram identificadas utilizando-se o Drugdex ${ }^{\circledR}(15)$, que juntamente com o Dynamed $\AA(16)$ e o bulário eletrônico da ANVISA (17) serviram para avaliar os demais aspectos relacionados à terapia, como a posologia, dosagem, efeitos adversos e recomendações. Os PRM foram classificados de acordo com Rovers e Currie (2010) com base na necessidade relacionada à terapia correspondente ao problema e a sua causa (18). Para identificação de medicamentos inapropriados para idosos, foram utilizados os critérios de 
Beers (19), e para avaliar a adesão à terapia farmacológica aplicou-se a metodologia adaptada de Morisky-Green (20).

\section{Intervenção Farmacêutica e Desfecho dos} casos. Após a análise inicial dos casos, foi programado e agendado o retorno de cada paciente. No retorno, o paciente foi orientado em relação à sua terapia farmacológica e foram realizadas as recomendações e intervenções farmacêuticas necessárias, buscando-se medidas para solucionar os PRM identificados. Os pacientes recebiam um material informativo personalizado, o qual apresentava recomendações a respeito dos medicamentos utilizados relacionando-se com sua condição clínica. Quando pertinente, os novos dados laboratoriais eram avaliados, a fim de observar os resultados obtidos a partir da intervenção realizada e a adesão do mesmo a tais medidas. Todas as intervenções e as demais informações eram registradas na ficha do paciente elaborada em uma planilha do Microsoft Excel.

\section{RESULTADOS E DISCUSSÃO}

Os participantes do estudo apresentaram idade média de $67 \pm 12$ anos, sendo $9(34,6 \%)$ do sexo masculino e $17(65,4 \%)$ do sexo feminino. A partir do IMC e da RCQ dos pacientes, foi verificada uma prevalência de participantes sobrepesos $(42,3 \%)$ e obesos $(30,8 \%)$, sendo que $65,4 \%$ dos pacientes encontravam-se sob risco muito alto de desenvolver doenças cardiovasculares (DCV) (Figura 2).

Entre os problemas de saúde relatados, o mais recorrente foi a hipertensão arterial, a qual acometia $80,8 \%$ dos participantes, seguida da hipercolesterolemia $(46,2 \%)$ e diabetes mellitus $(38,5 \%)$, sendo que $57,7 \%$ dos pacientes apresentavam combinação de duas ou três destas condições clínicas. Além disso, 61,5\% dos pacientes relataram outra doença cardiovascular, como: arritmia (31,2\%), aterosclerose $(18,8 \%)$, infarto agudo do miocárdio $(18,8 \%)$, insuficiência cardíaca $(12,5 \%)$, transtornos do aparelho circulatório (12,5\%), e acidente vascular cerebral $(6,2 \%)$.

As doenças crônicas são apontadas como os principais fatores de risco para desenvolvimento de doenças cardiovasculares $(21,22,23,24)$, as quais constituem as principais causas de morte no mundo nos últimos 15 anos (25). A ocorrência de múltiplas condições clínicas também é a principal causa de polimedicação, pois para cada uma destas enfermidades, torna-se necessária a associação de fármacos para se alcançar um tratamento farmacológico eficaz, situação que está diretamente relacionada ao aumento da probabilidade de PRM $(24,26,27)$.

Figura 2. Perfil antropométrico dos pacientes avaliados em seguimento farmacoterapêutico realizado em uma farmácia escola na cidade de Campo Mourão, PR (2016-2017).

\section{Perfil dos participantes}

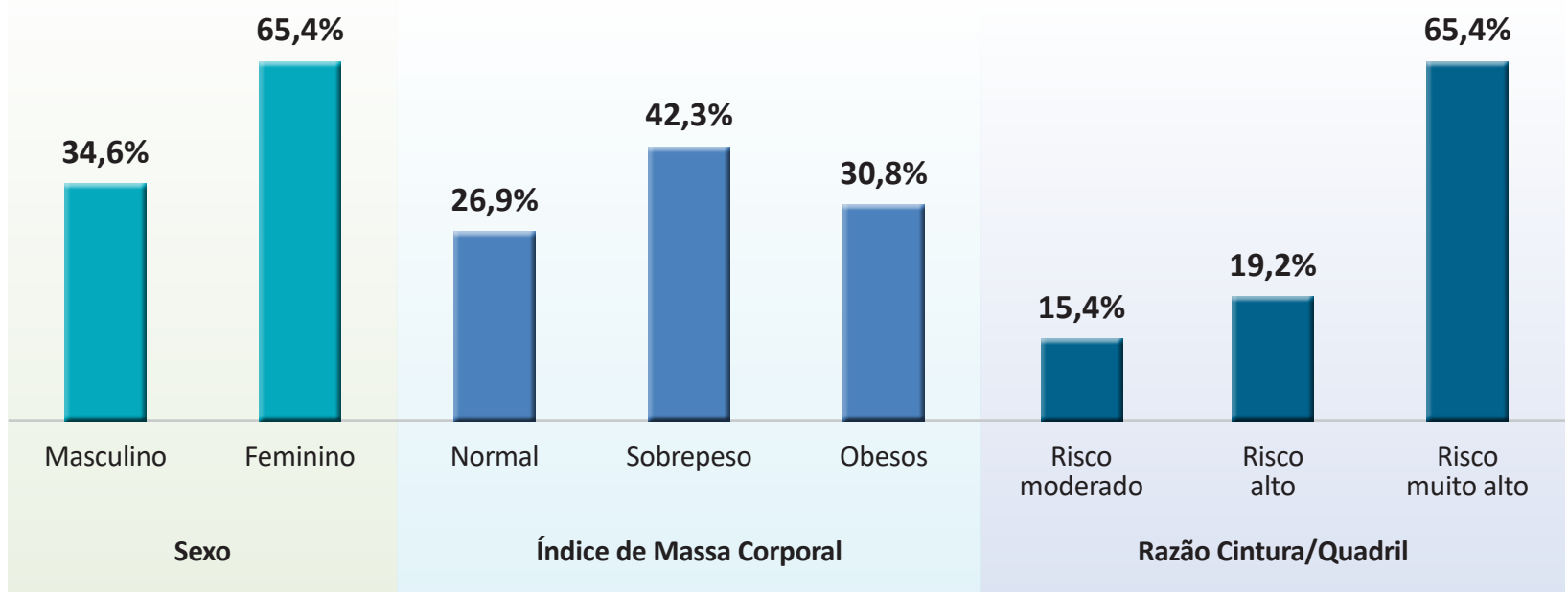


Referente ao tratamento farmacológico, cada paciente fazia uso de $8 \pm 2,4$ medicamentos. A polimedicação está diretamente associada ao avanço da idade, visto que a maioria dos pacientes avaliados eram idosos $(80,8 \%)$ e portador de três ou mais enfermidades.

No total, foram realizadas as análises e classificação de 222 medicamentos. Com base na classificação ATC, observou-se uma prevalência de medicamentos que atuam no sistema cardiovascular ( $\mathrm{C}=37,4 \%$ ), seguido dos que atuam no aparelho digestivo e metabolismo $(A=23,9 \%)$ e na circulação sanguínea $(B=12,2 \%)$ (Tabela 1$)$.

No presente estudo, foram observados, em média, quatro PRM por paciente. Na literatura, a incidência destes problemas varia bastante conforme a população, de modo que alguns estudos apresentam uma ocorrência de um PRM a cada um a cinco pacientes $(21,28)$, enquanto outros apresentam dois a três PRM a cada paciente analisado $(26,29,30)$.

Foram identificados 108 PRM, sendo 44 problemas referentes à segurança, 38 à efetividade, 10 à necessidade de terapia adicional, 9 à tratamento desnecessário e 0 à não adesão ao tratamento (Figura 3). O PRM prevalente foi o de reações adversas $(27,8 \% ; 30 / 108)$, que se enquadra no
Tabela 1. Classificação dos medicamentos prescritos aos pacientes submetidos a seguimento farmacoterapêutico realizado em Campo Mourão, PR, no período de 2016-2017, de acordo com a classificação ATCC (Anatomical Therapeutic Chemical Code).

\begin{tabular}{|l|c|c|}
\hline \multicolumn{1}{|c|}{ Classe } & Quantidade & Fármacos (\%) \\
\hline $\begin{array}{l}\text { C. Sistema cardiovascular } \\
\text { A. Aparelho digestivo e } \\
\text { metabolismo }\end{array}$ & 83 & $37,4 \%$ \\
\hline B. Circulação sanguínea & 53 & $23,9 \%$ \\
\hline N. Sistema nervoso & 27 & $12,2 \%$ \\
\hline $\begin{array}{l}\text { M. Sistema } \\
\text { musculoesquelético }\end{array}$ & 16 & $11,3 \%$ \\
\hline H. Preparações hormonais \\
sistêmicas
\end{tabular}

Figura 3. Classificação dos PRM identificados nos pacientes submetidos ao seguimento farmacoterapêutico realizado em Campo Mourão, PR, no período de 2016-2017, de acordo com a necessidade referente à terapia farmacológica.

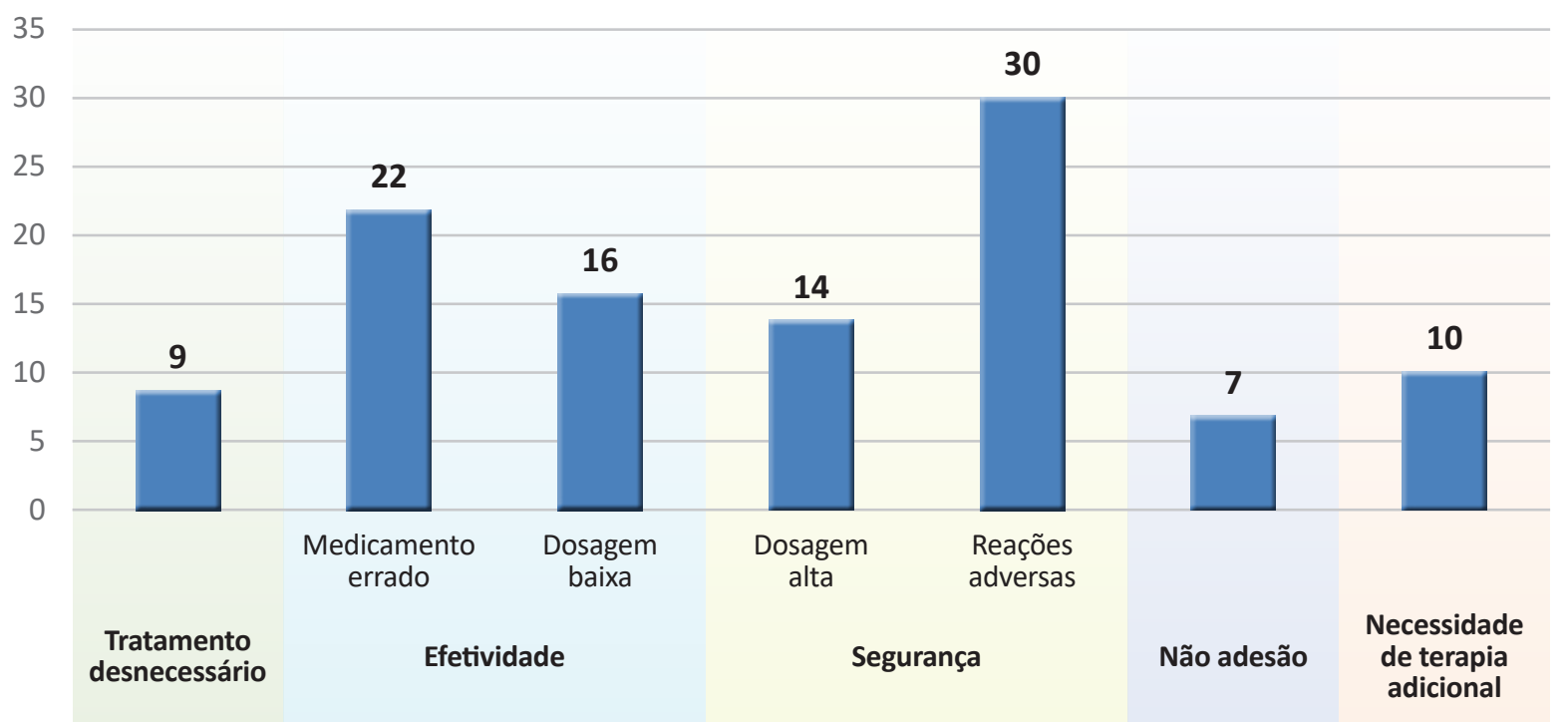


quesito segurança do tratamento. Este resultado diverge dos obtidos por outros estudos, os quais relataram prevalência de PRM relacionados à necessidade de terapia adicional (31), à efetividade da terapia $(26,28)$ e a segurança da terapia $(30)$.

As interações medicamentosas destacaram-se como as principais causas de PRM (30,5\%; 33/108), das quais $14(42,5 \%)$ eram interações medicamentosas potenciais associadas ao risco de reações adversas e as $19(57,5 \%)$ relacionadas à dosagem dos medicamentos.

Ainda referente a questões relacionadas à segurança da terapia medicamentosa, $57,7 \%$ dos idosos tiveram medicamentos classificados como potencialmente inapropriados a idosos de acordo com o critério de Beers (19). Tal condição foi considerada um problema relacionado à segurança da terapia, correspondendo a 13,9\% dos PRM encontrados no estudo, e a $34,1 \%(15 / 44)$ dos PRM referentes a segurança. Esses medicamentos podem acarretar em eventos adversos a saúde do paciente, podendo ocasionar complicações como fraturas, déficit cognitivo, depressão, sangramentos gastrointestinais, disfunção renal, como também agravar quadros de insuficiência cardíaca congestiva (32).

Dos medicamentos potencialmente inapropriados para idosos, $35,0 \%$ correspondiam ao fármaco omeprazol, o qual está relacionado ao aumento do risco de osteoporose/fraturas, infecção por Clostridium difficile, atrofia gástrica, como também redução da absorção de vitamina B12, ocasionando assim danos ao sistema nervoso, o que pode resultar em quadros de demência (33). É recomendado que o uso de medicamentos inibidores da bomba de prótons (IBP) sejam evitados em pacientes com mais de 65 anos de idade, e caso necessário, o tratamento seja inferior a oito semanas.

No entanto, o uso excessivamente prolongado destes medicamentos tem aumentado principalmente entre pacientes idosos $(34,35)$. Em um estudo realizado por Oliveira e cols. (2017) foi mostrada uma prevalência de 1.220 medicamentos potencialmente inapropriados prescritos para idosos, dos quais $39,4 \%$ pertenciam à classe dos IBP (33).

Outro problema recorrente entre os pacientes foi a não adesão à terapia farmacológica, visto que $46,3 \%$ (12/26) dos participantes relataram, em algum momento do acompanhamento, não fazer uso de um ou mais dos medicamentos prescritos. Dentre as justificativas para a não adesão, destacaram-se as queixas referentes à quantidade de medicamentos utilizados. Também foi observada a falta de compreensão dos pacientes em relação à terapia prescrita, visto que, ao serem questionados sobre o esquema terapêutico seguido, apresentaram dúvidas em relação aos medicamentos e por diversas vezes confundiam medicamentos com a cor ou formato semelhante. Este problema compromete significativamente a eficácia do tratamento farmacológico, resultando no agravo da condição clínica e podendo acarretar em hospitalizações ou complicações no quadro clínico do paciente (18).

Foram realizadas 76 intervenções farmacêuticas referentes à terapia farmacológica em 17 $(65,4 \%)$ dos pacientes. Os demais participantes não retornaram para a segunda fase do acompanhamento. Dentre as intervenções realizadas, três indivíduos necessitaram de alteração na prescrição para substituição de terapia. Neste caso, foi encaminhada uma carta ao médico prescritor, na qual era descrito o caso do paciente e a justificativa para alteração da terapia farmacológica.

Estima-se que $60,5 \%$ das intervenções foram aderidas pelos pacientes, mas este dado pode ser considerado subjetivo, uma vez que tal proporção é baseada principalmente no relato do paciente no momento da consulta.

É notório que por meio de um acompanhamento regular, seja diário ou semanal, pode-se obter resultados mais satisfatórios relacionados à intervenção farmacêutica. Em ambientes como clínicas e hospitais, este acompanhamento torna-se mais possível. Em um estudo realizado em um hospital universitário de São Paulo (23), o índice de adesão às intervenções propostas pelo farmacêutico clínico foi de $71,5 \%$, o qual se aproxima de um estudo realizado também em um hospital universitário do Paraná, no qual houve $76,3 \%$ de aceitação das intervenções propostas pelo profissional (11).

Apesar da crescente discussão acerca do tema, ainda não há muitos estudos que avaliem a prevalência de PRM em pacientes polimedicados não internados, o que dificultou a comparação dos dados obtidos neste seguimento, sendo que a maior parte dos trabalhos acerca do tema referem-se a pacientes hospitalizados e ambulatoriais. 
Neste contexto, torna-se evidente a necessidade de prevenir e corrigir a ocorrência de PRM, garantindo ao paciente um tratamento seguro e eficaz. Para isto, é de suma importância a participação ativa do farmacêutico clínico no seguimento farmacoterapêutico, a fim de identificar, solucionar e prevenir a ocorrência de PRM e agravos na saúde. Cabe ao profissional farmacêutico oferecer aos pacientes informações e os cuidados necessários para que os mesmos compreendam seu tratamento e possam cumpri-lo de maneira correta, efetiva e segura.

\section{CONCLUSÃO}

Dentre os fatores que podem ser apontados como predisponentes para a incidência de PRM, destacam-se a polimedicação, a idade avançada e a presença de doenças crônicas. A maioria dos PRM estava associada à segurança e à efetividade, dentre as causas identificadas destacou-se o uso de medicamentos classificados como inapropriados a pacientes idosos e a não adesão à terapia, respectivamente.

\section{REFERÊNCIAS}

1. Masnoon N, Shakib S, Kalisch-Ellett L, Caughey GE. What is polypharmacy? A systematic review of definitions. BMC Geriatr. 2017;17(1):230-240. DOI: 10.1186/s12877-017-0621-2.

2. Wuyts J, Maesschalck J, De Wulf I, Foubert K, Boussery K, De Lepeleire J, Foulon V. Studying the impact of a medication use evaluation for polymedicated older patients by the community pharmacist - study protocol. BMC Health Serv Res. 2018;18(1):623-631. DOI: 10.1186/s12913-018-3440-z.

3. Pereira KG, Peres MA, Iop D, Boing AC, Boing AF, Aziz M, D’Orsi E. Polifarmácia em idosos: um estudo de base populacional. Rev Bras epidemiol. 2017;20(2):335-344. DOI: $10.1590 / 1980-5497201700020013$.

4. Macinko J, Andrade FB, Souza-Junior PRB, Lima-Costa MF. Primary care and healthcare utilization among older Brazilians. Rev Saúde Pública 2018;52(2):6s. DOI: 10.11606/S1518-8787.2018052000595.

5. IBGE.-- Pesquisa Nacional de Saúde: percepção do estado de saúde, estilos de vida e doenças crônicas: 2013. Instituto Brasileiro de Geografia e Estatística. Rio de Janeiro: IBGE; 2014.

6. Hernández DS, Castro MMS, Dadér MJF. Método Dáder: Manual de seguimento farmacoterapêutico. Espanha: Granada. 2009. 128 p.

7. Correr CJ. Os problemas relacionados aos medicamentos no contexto da atenção farmacêutica: Uma avaliação de conceitos. Infarma - Cienc Farmac. 2002;14(5/6):73-78.

8. Ebbesen J, Buajordet I, Erikssen J, Brors O, Hilberg T, Svaar H, Sandvik L. Drug-related deaths in department of internal medicine. Arch Intern Med. 2001;161(19): 2317-2323. DOI: 10.1001/archinte.161.19.2317.

9. Capucho HC. Monitoramento e avaliação farmacoterapêutica: O medicamento faz efeito? Qual? Brasília: OPAS/OMS; 2016.
10. Kaboli PJ, Hoth AB, Mcclimon BJ, Schnipper JL. Clinical pharmacists and inpatient medical care: A systematic review. Arch Intern Med. 2006;166(9): 955-964. DOI: 10.1001/archinte.166.9.955

11. Reis WCT, Scopel CT, Correr CJ, Andrzejevski VMS. Análise das intervenções de farmacêuticos clínicos em um hospital de ensino terciário do Brasil. Einstein 2013;11(2):190-196. DOI: 10.1590/S1679-4508201300 0200010 .

12. SBC. Sociedade Brasileira de Cardiologia. VII Diretrizes brasileiras de hipertensão. Arq Bras Cardiol. 2016;107(3):1-83.

13. SBD. Diretrizes da Sociedade Brasileira de Diabetes: 2017-2018. Sociedade Brasileira de Diabetes. São Paulo: Clannad. 2017.

14. BRASIL. Orientações para coleta e análise de dados antropométricos em serviços de saúde: norma técnica do sistema de Vigilância Alimentar e Nutricional - SISVAN. Brasília: Ministério da Saúde. 2011.

15. Drugdex System [Internet]. Greenwood Village: Thomson MICROMEDEX; 1974-2006. Disponível em: http:/www.periodicos.capes.gov.br.

16. DynaMed [Internet]. Ipswich: EBSCO Information Services. 1995. Disponível em: http://www.www. dynamed.com.

17. ANVISA. Bulário eletrônico [Internet]. Agência Nacional de Vigilância Sanitária. Disponível em: https://consultas. anvisa.gov.br/\#/bulario/.

18. Rovers JP, Currie JD. Guia prático da atenção farmacêutica: manual de habilidades clínicas. 1st ed. São Paulo: Pharmabooks. 2010.

19. AGS. American Geriatrics Society. 2019 Updated AGS Beers Criteria ${ }^{\circledR}$ for Potentially Inappropriate Medication Use in Older Adults. J Am Geriatr Soc. 2019; 67(4): 674-694. DOI: 10.1111/jgs.15767. 
20. Morisky DE, Green LW, Levine DM. Concurrent and predictive validity of a self-reported measure of medication adherence. Med Care 1986;24(1):67-74. DOI: 10.1097/00005650-198601000-00007.

21. Medeiros Netto AS, Melo FB, Silva WB. Frecuencia de problemas relacionados com los medicamentos en pacientes que visitarion el servicio de urgencia de un hospital regional. Seg Farmac. 2005;3(1):213-224.

22. Chehuen-Neto JA, Delgado AAA, Duarte Galvão CCG, Machado SJM, Bicalho TC, Oliveira TA. Uso de medicamentos por idosos de Juiz de Fora: um olhar sobre a polifarmácia. HU Rev. 2012; 37(3):305-313.

23. Viana SSC, Arantes T, Ribeiro SCC. Intervenções do farmacêutico clínico em uma Unidade de Cuidados Intermediários com foco no paciente idoso. Einstein 2017;15(3):283-288. DOI: 10.1590/S1679-45082017 AO3894

24. BRASIL. Hipertensão arterial sistêmica (HAS) e Diabetes mellitus (DM): protocolo. Brasília: Ministério da Saúde. 2001. Disponível em: http://bvsms.saude.gov. br/bvs/publicacoes/cd05_06.pdf.

25. OMS. Dez principais causas de morte no mundo. Folha informativa: OPAS/OMS. Organização mundial da saúde. 2018. Disponível em: https://www.paho.org.

26. Provin MP, Campos AP, Nielson SEO, Amaral RG. Atenção Farmacêutica em Goiânia: inserção do farmacêutico na Estratégia Saúde da Família. Saúde Soc. São Paulo 2010;19(3):717-723.

27. Galato D, Silva ES, Tiburcio LS. Estudo de utilização de medicamentos em idosos residentes em uma cidade do sul de Santa Catarina (Brasil): um olhar sobre a polimedicação. Cien Saude Colet. 2010;5(1):2899-2905. DOI: $10.1590 / \mathrm{S} 1413-81232010000600027$.

28. Janebro DI, Belém LF, Tomaz ACA, Pinto DS, Ximenes LMA. Problemas Relacionados aos Medicamentos (PRM's) em Pacientes Pediátricos de um Hospital no Município de Campina Grande, Paraíba, Brasil. Lat Am J Pharm. 2008;27(5):681-687.
29. Garcia NI, Jané CC, Creus MT, Dalmau LM, Vila RG, Sala JB. Evaluación de la integración del farmacêutico em equipos de atención de unidades de hospitalización. Farm Hosp. 2002;26(1):18-27. DOI: 10.1590/S167704202003000300006.

30. Nunes LMN, Lopes NMS, Fonteles MMF. Acompanhamento farmacoterapêutico de pacientes diabéticos tipo 2 e fatores de risco associados. Rev Bras Farm. 2012;93(2):196-203.

31. Lima APD, Rios AD, Shimokawa Borges JSS, Ferreir JM, Ferreira RB, Cunha CRM. Consulta farmacêutica e análise de problemas relacionados à medicação em um hospital da regional oeste do estado de Goiás. Rev eletron FMB. 2013;6(1):1-15.

32. BRASIL. Instituto para práticas seguras no uso de medicamentos - ISMP. Medicamentos potencialmente inadequados para idosos. Boletim ISMP. 2017;7(3). Disponível em: https://www.ismp-brasil.org/site/wpcontent/uploads/2017/09/is_0006_17a_boletim_agosto_ ismp_210x276mm_v2.pdf.

33. Oliveira HSB, Sousa JRP, Donis ACG, Manso MEG. Utilização dos critérios de Beers para avaliação das prescrições em idosos portadores de doenças crônicas vinculados a um plano de saúde. RBCEH. 2017;14(3): 242-251. DOI: 10.5335/rbceh.v14i3.7376.

34. Lima JM, Dal Fabbro AL, Funayama AR. Uso do omeprazol: estudo descritivo de pacientes idosos de uma unidade de saúde da família (USF) de Ribeirão Preto, SP, Brasil. Infarma - Cienc Farmac. 2019;31(1):46-53. DOI: 10.14450/2318-9312.v31.e1.a2019.pp46-53.

35. Pimenta LR, Soares RS, Castro PFS, Freitas JGA, Nielson SEO. Uso indiscriminado de omeprazol em idosos e a importância da atenção farmacêutica. Universo 2016;3(1):303-318. 